\title{
ON THE DISTANCE TO THE OPEN CLUSTER LYNG尺 6
}

E. R. Anderson, B.F. Madore and M.H. Pedreros

Dept. of Astronomy, Univ. of Toronto, Toronto, Ontario

The determination of the membership of the Cepheid TW Normae to the galactic cluster Lynga 6 is important because TW Nor would then be the intrinsically brightest Cepheid associated with a cluster.

A series of nine photographic exposures (three each in $\mathrm{U}, \mathrm{B}$ and $\mathrm{V}$ ) were obtained during open time at the Anglo-Australian Telescope. The plates were measured with an iris photometer and the data calibrated using the photoelectric standards of van den Bergh and Harris (1976). The data for 300 stars were then analysed using a new computerized method of main sequence fitting and cluster membership determination developed by Pedreros (1984). We found 41 member stars with a mean reddening $E(B-V)=1.33^{ \pm}=0.10$ and a distance modulus of 11.1 \pm 0.4 magnitudes.

Figure 1 is a photo of the cluster with the member stars labelled. Figure 2 shows the colour-magnitude diagram for the cluster. The solid line is the zero age main sequence drawn for an assumed distance modulus of 11.1. The Cepheid TW Nor is represented by a solid triangle. Also shown is an evolved G type star (star L) believed by both Madore (1975) and Lynga (1977) to be a cluster member.

The infrared period-luminosity relation and observations of TW Nor (Welch et al. 1984b, 1984a) yield a distance modulus of $11.8 \pm 0.2$. The dashed line in Figure 2 is the zero age main sequence drawn for this assumed distance. Since the infrared Cepheid distance scale appears to be well established, we are then left with two possible conclusions: 1.) Lynga 6 is at a distance modulus of 11.1 and TW Nor is not a member but rather behind the cluster at a distance modulus of 11.8. or, 2.) The Cepheid distance is the correct distance to the cluster. The stars we have assumed to be main sequence stars are actually slightly evolved B stars and/or the reddening is wrong. REFERENCES

van den Bergh, S. and Harris, G. 1976, Ap.J., 208, 765

Lynga, G. 1977, Astron. and Astrophys., 54, 311.

Madore, B.F. 1975, Astron. and Astrophys., 38, 471.

Pedreros, M.H. 1984, Ph.D. Thesis, University of Toronto.

Welch, D.L., Wieland, F., McAlary,V.W., McGonnegal, R., Madore, B.F., McLaren,R.A., and Neugebauer,G. 1984a, Ap.J. Supp., 54, 547.

Welch,D.L., McAlary,C.W., Madore,B.F., McLaren, R.A. and Neugebauer, G. 1984b, In preparation. 


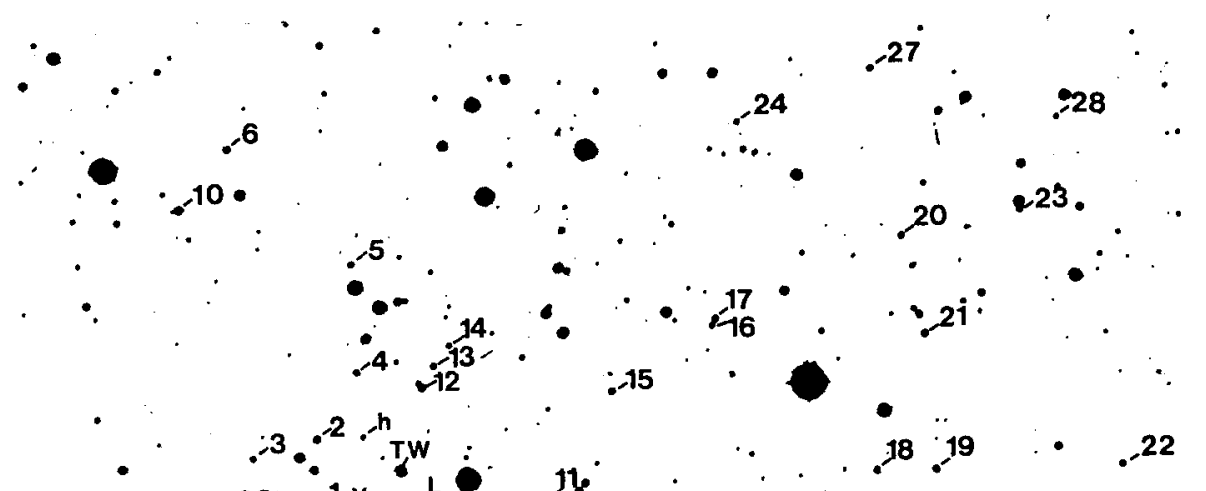
30 . 35 .

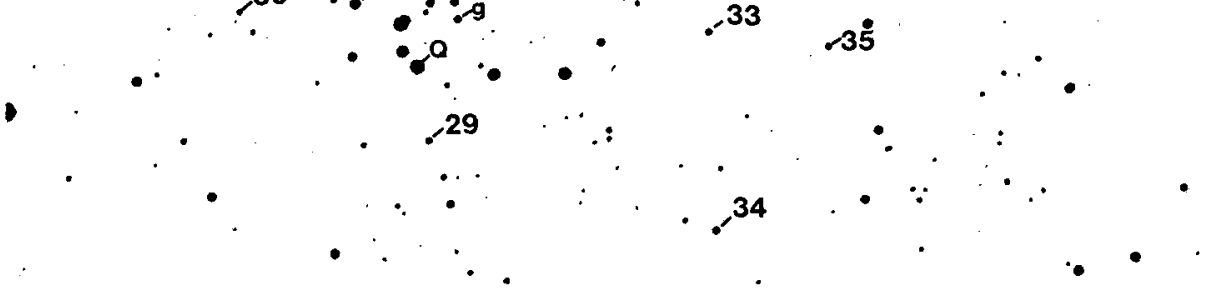

FIGURE 1. The open cluster Lyngå 6. (U photo)

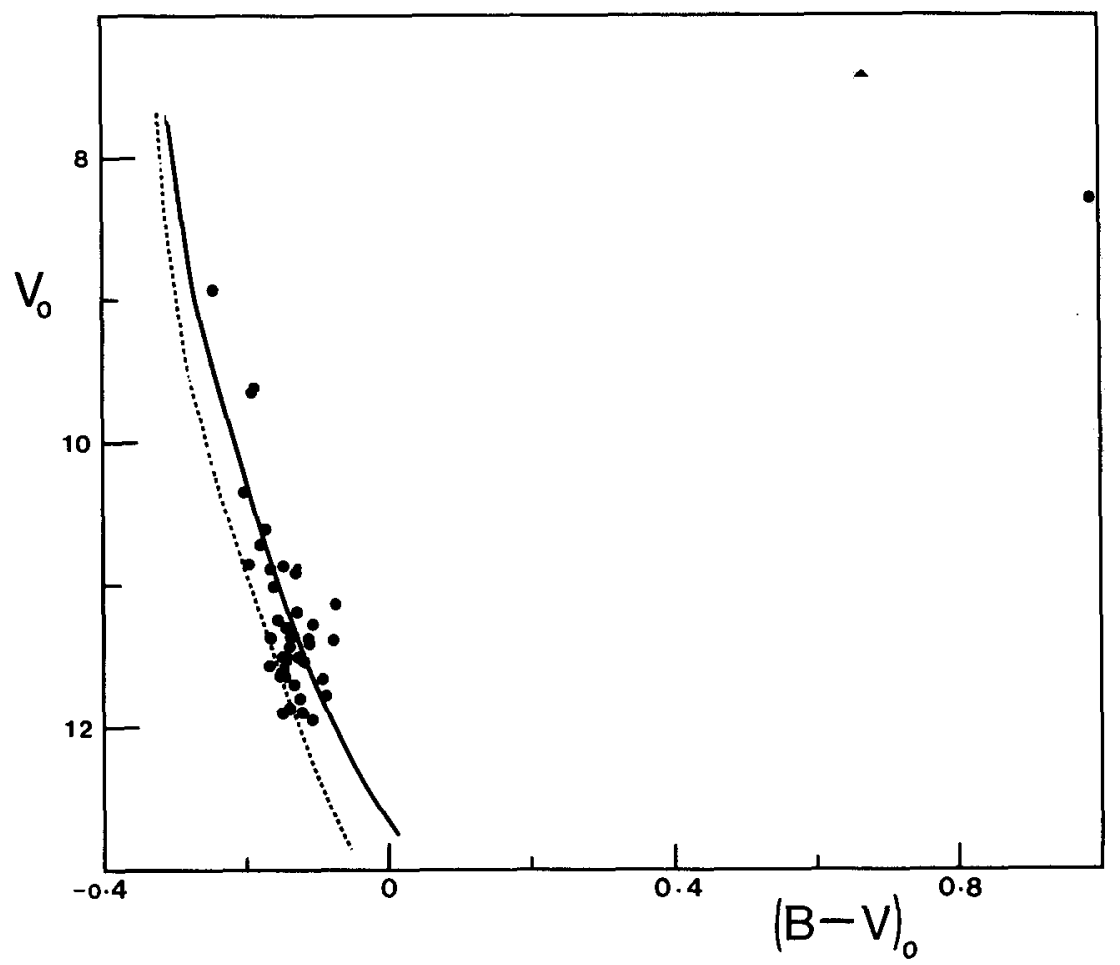

FIGURE 2. The colour-magnitude diagram for Lyngå 6 . 